\title{
The importance of networking
}

Remember cloning a gene the oldschool way? Or studying only one gene at a time? A recent paper by Quigley et al. is the latest in a series of studies to apply the construction of genetic networks to linking genotypes to phenotypes, specifically in mice. The results highlight the potential of this approach for identifying genes with key roles in phenotypes that are related to disease.

To identify genetic motifs linked to skin cancer and inflammation, Quigley and colleagues crossed mice

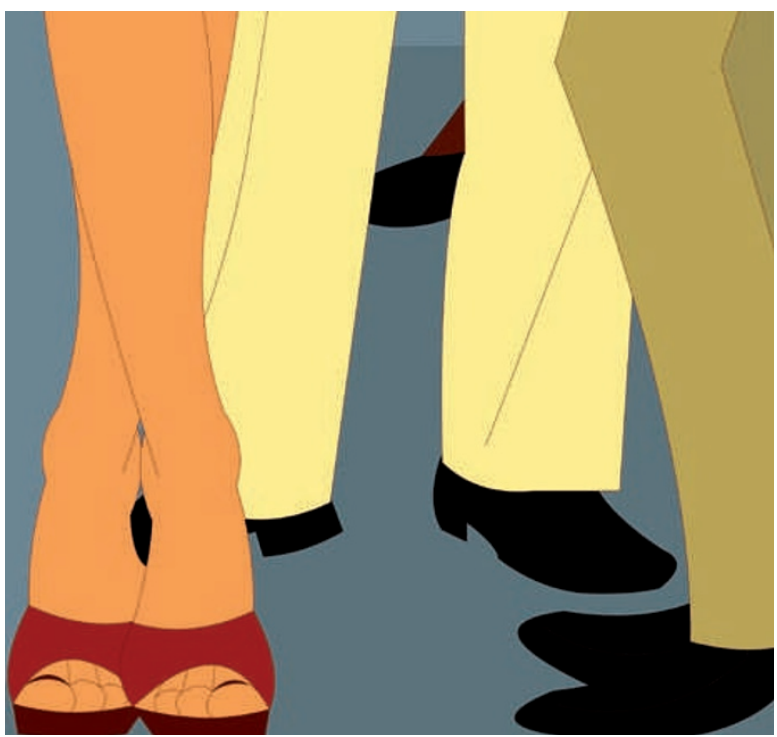

of two species, Mus spretus and Mus musculus, as they are respectively resistant and susceptible to tumour development. After inducing skin tumours in a number of the mice, they extracted mRNA from uninvolved tail skin for gene expression analysis. Combining mRNA profiling with linkage analysis allowed the authors to construct a 'susceptibility network' of gene expression and regulation in the normal skin.

The normal skin network identified at least 62 genes involved in hair follicle biology, which are regulated by numerous expression QTLs (eQTLs; genetic loci that influence other genes in cis or in trans). A candidate regulatory gene in the hair follicle network was the G-proteincoupled receptor $\underline{\operatorname{Lg} r 5}$, agreeing with its previously described role as a stem cell marker in hair follicles and intestinal cells. Further work is needed to determine whether Lgr5 is the 'master regulator' of hair follicles; presumably this would be of great interest to industries concerned with hair regrowth.

Construction of the gene expression networks from skin tumour-susceptible and resistant mice suggested that normal skin from susceptible mice showed enriched expression of genes involved in inflammation as well as in cell growth and its regulation. One locus on mouse chromosome 15 seems to be linked to expression of numerous genes in a network for inflammation and barrier function. The researchers focused on $\underline{V d r}$ as the best candidate master regulator of this network. $V d r$ encodes the mouse vitamin D receptor, and vitamin D levels in humans have been linked to cancer susceptibility in previous studies. The authors speculate that several sequence changes between the $M$. spretus and M. musculus alleles affect the protein's function and resulting tumour susceptibility. These results support the notion that adequate levels of vitamin $\mathrm{D}$ are important for health, as well as confirming the power of the genetic network approach for identifying genotype-phenotype relationships.

Chris Gunter, HudsonAlpha Institute for Biotechnology

ORIGINAL RESEARCH PAPER Quigley, D. A. et al. Genetic architecture of mouse skin inflammation and tumour susceptibility. Nature 11 Jan 2009 (doi:10.1038/nature07683) WEB SITE

Balmain laboratory homepage: http://cancer.ucsf.edu/balmain/index.php 